\title{
The Use of Unmanned Aircraft System for the Radio Frequency Interference Measurements
}

\author{
Marjo Heikkilä, Arto Seppänen, \\ Marjut Koskela \\ Centria University of Applied Science \\ Ylivieska, Finland \\ Email:marjo.heikkila@centria.fi
}

\author{
Jukka Pihonen, Jan Engelberg \\ The Finnish Transport and Communications Agency \\ Traficom \\ Helsinki, Finland \\ Email: jukka.pihonen@traficom.fi
}

\author{
Ari Pouttu \\ University of Oulu \\ Oulu, Finland \\ Email: ari.pouttu@oulu.fi
}

\begin{abstract}
The unmanned aircraft systems (UAS) are commonly used for aerial photography, express delivery for packets or video monitoring. The UAS technology can help responding to the growing demands of the frequency monitoring by national radiocommunication authorities (NRAs). This article presents a practical usage trial of an unmanned aircraft for monitoring the spectrum usage and finding the interference location. The monitoring of spectrum usage is one part of the NRAs' process for guarantee user compliance with radio license conditions. Finding illegal or malfunctioning transmitters is a challenging task especially in urban environment, due to multi-path propagation conditions. This study focuses on practical assessment of the method for determining accurate location of radio frequency interference sources from the buildings using the UAS technology. The practicability of the UAS technology usage in spectrum monitoring activities was under the evaluation. This study showed promising results that UAS with the measurement equipment setup suits NRAs' as a tool for mobile monitoring on the air.

Index Terms-Field trials, RF Interference Measurements, Spectrum monitoring, Unmanned Aircraft System, Unmanned Aerial Vehicle
\end{abstract}

\section{INTRODUCTION}

This paper depicts and assesses an interference measurement method for wireless networks. Wireless systems use electromagnetic waves, mainly radio waves, to transmit data over the transmission channel. The predictions of wireless communications especially in mobile communications shows that the amount of traffic will grow globally [1]. The emerging mobile communication system called 5G will serve several types of end users and offers services in new frequency bands [2]. One of the new types of communication of $5 \mathrm{G}$ is machine type communications, i.e. when intelligent machines are utilizing mobile broadband or upcoming $5 \mathrm{G}$ massive machine type (5G-mMTC) services. The interference-free radio communication and the efficient use of radio spectrum is essential to all radio systems. The radio spectrum is economically remarkable resource for nations itself. International and national regulation are needed to oversee and control the use of these resources. Radio frequencies are allocated to radiocommunication services (e.g. mobile service, fixed service, various satellite services, scientific use etc.). The Ministry of Transport and Communications is responsible for frequency policy in Finland. The Finnish Transport and Communications Agency Traficom works under the Ministry of Transport and Communications. Traficom grants radio licenses, guides and supervises the use of radio frequencies. The Finnish frequency regulation, so called The National Table of Frequency Allocations, covers frequency range between $8.3 \mathrm{kHz}-400 \mathrm{GHz}$ [3].

Radio frequency systems are vulnerable and susceptible to interference. When radio frequencies are used without approval or outside the legal and regulatory conditions it could cause interference. Radio devices can cause disturbances to each other if they operate in the same geographical area and utilize the same frequency range. They can interfere, decrease capacity or quality of the transmission. The frequency regulation is aiming to create the good conditions for wireless systems, but there are still problems to be solved. Many phenomena can cause radio frequency inferences like radio equipment itself or other electric devices (such as LED lights) or the phenomena of nature (Aurora Borealis, harsh wind conditions and so on). Faulty systems, even receiver systems may cause interference. Traficom guides and monitors the usage of radio frequencies but also solves radio frequency interference cases. They perform additional measurements for finding interference sources. National Regulatory Authorities get massive amount of announcements on problems related to the use of radiocommunication systems. In the year 2014 FICORA (Traficom since 1.1.2019) [4] has cleared up to 120 radio disturbances. Most of these disturbances were originating from domestic TV-antenna amplifiers. Almost 15 percent of the cases were due to malfunctioning electronics. These types of radio interferences cause a need for field measurement work for NRAs. 41 The European Conference of Postal and Telecommunications Administrations (CEPT) countries reported 21.527 interference cases in the year 2017 [5]. The most common sources of interference were public mobile networks, illegal radio devices and electric appliances. In most cases the interference source was unknown.

The radio frequency monitoring for solving interference cases has been conducted by mobile RF measurement setup which is build in a car. There is also a need for hand-held setup when performing RF measurements by walking. These are useful methods, however, the new UAS technology is suitable for professional operation to support existing methods. The interest to use of UAS technology as a part of frequency 
monitoring is increasing in many countries. There can be many benefits for using UAS in frequency monitoring. The UAS technology brings new possibilities to perform monitoring in hard-to-reach places. It is seen that usage of UAS could increase the efficiency of frequency monitoring (e.g. less time spent at the field). This study evaluates the practicability of the UAS technology in spectrum monitoring activities. The study describes one measurement method for spectrum monitoring measurements with UAS.

The Section I introduces a background and a need for interference measurement. The related work is described in Section II. The measurement setup with measurement environment and equipment are presented in Section III. The results are shown in Section V. The conclusions are drawn and future work is discussed in Section VI.

\section{RELATED WORK}

The monitoring of spectrum usage is needed to guarantee user compliance with license conditions. Authorities perform monitoring with fixed, remote and mobile monitoring stations. All of these methods are also helpful to identify sources of interference and locating legal and illegal transmitters. Determining the location of interference source with Radio Direction-Finding (RDF) method is typically based on the mobile monitoring stations [6]. The mobile monitoring station is built in the car. It includes radio receivers, spectrum analysers, amplifiers, direction-finding equipment and a directional antenna. Adjustable antenna mast structure is also needed in the mobile monitoring station to increase the received signal level.

The receiving antenna is an essential component of the monitoring station to receive radio waves. Antenna type must be selected according to requirements for the frequency range and the required need of directionality. The overall spectrum scans on the surrounding environment are usually performed with omni-directional antennas. When there is a need to identify the direction of the interference source, the antenna must have high directivity in the certain direction. Due to directivity the power radiated from other directions are negligible.

A spectrum analyser is used to make interference measurement. It is used to analyse the content of spectrum. RF frequency range, resolution and sensitivity are important characteristics of the spectrum analyser.

In the RDF method directional antenna is rotated to measure the signal strength of the interfering signal as a function of the direction. When measurements are repeated in the different locations, the direction of the most powerful signals can give the exact location of the interference source. The use of this method is problematic especially in urban environment because of multi-path propagation. Signals can reflect from high buildings and other obstacles in a way that the strongest signal comes from the opposite direction than the original interference source location.

One solution to tackle these challenges is to perform measurements in line-of-sight environment with using the unmanned aircraft system (UAS). Duthoid et al. [3] introduced the in-situ antenna characterization measurements of X-band at $9.8 \mathrm{GHz}$ [7]. Garc et al. [8] introduced antenna array measurements in near field with UAS. Heikkilä et al. [9] presents study of the usage of UASs for site survey and antenna pattern measurements in-situ in mobile networks. These references showed the usability of UAS in the site survey measurements. When UAS is used in RDF measurement, the unwanted impact of the reflections can be reduced by executing RF measurements up above the roof tops. This can be implemented by fastening the spectrum analyser and measurement antenna to UAS. The drone with the measurement setup can fly over different terrain obstacles.

National authorities in many countries are interested in using UAS for example in performing radio frequency interference (RFI) measurements or the localisation of jamming sources. The National Frequencies Agency of France (ANFR) [10] has considered benefits, constraints and the regulatory framework of using UAS in spectrum monitoring. ANFR have performed the experiments of site survey and electromagnetic field measurements with UAS. They were interested in the UASs ability to perform RFI measurements in hard-to-reach places.

\section{MeAsurement Setup}

The study was performed in the suburban environment of Ylivieska, Finland. A four floor flat was selected for measurement location (Fig. 1.). The interference source was located on the fourth floor of the building (Fig. 2.).

The measurement setup describes the components used in the reference measurements on the ground and in the air (Table I) measurements.

\section{A. Source of interference}

The inference sources are varying case by case, but some typical sources are malfunctioning electronic devices. For this reason wireless microphone AKG HT 40 MiniPro was selected as an interference source. The transmission frequency of the microphone was $864.850 \mathrm{MHz}$ and the transmission power was $10 \mathrm{~mW}$.

\section{B. Unmanned Aircraft System}

The commercial Unmanned Aerial Vehicle (UAV) DJI S900 Speading Wings was used in the study. It has suitable performance for this type of operation. It has enough payload capacity to carry measurement setup. The size of the UAV is following: diagonal wheelbase $900 \mathrm{~mm}$ and total height $730 \mathrm{~mm}$. In some case the battery lifetime is limiting the

TABLE I. TEST DEVICES

\begin{tabular}{|c|c|c|}
\hline Role & Equipment & Model \\
\hline UAS & $\begin{array}{c}\text { UAV } \\
\text { Ground Station SW } \\
\text { Ground Station HW } \\
\text { Remote Controller }\end{array}$ & $\begin{array}{c}\text { Drone DJI S900 } \\
\text { UgCS Desktop application } \\
\text { Laptop Computer } \\
\text { Futaba T14SG }\end{array}$ \\
\hline $\begin{array}{c}\text { Spectrum } \\
\text { Analyser }\end{array}$ & $\begin{array}{c}\text { Hand-held Aaronia } \\
\text { Spectran }\end{array}$ & HF-6105 \\
\hline Antenna & $\begin{array}{c}\text { Directional logarithmic- } \\
\text { periodic antenna }\end{array}$ & Aaronia HyperLOG 60100 \\
\hline
\end{tabular}




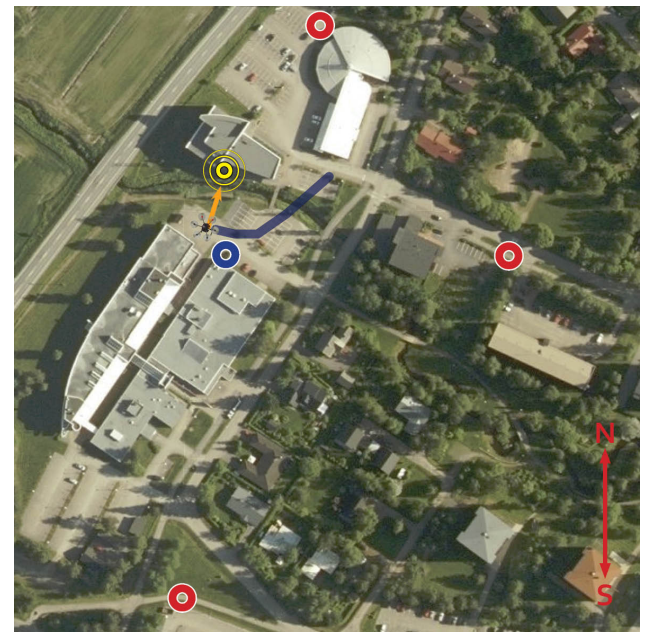

Figure 1. The aerial picture of the measurement environment. The RF source is marked with yellow, measurement location with blue circle and flying route for preameasurement with blue line. The locations of a triangle measurement are marked with red circles.

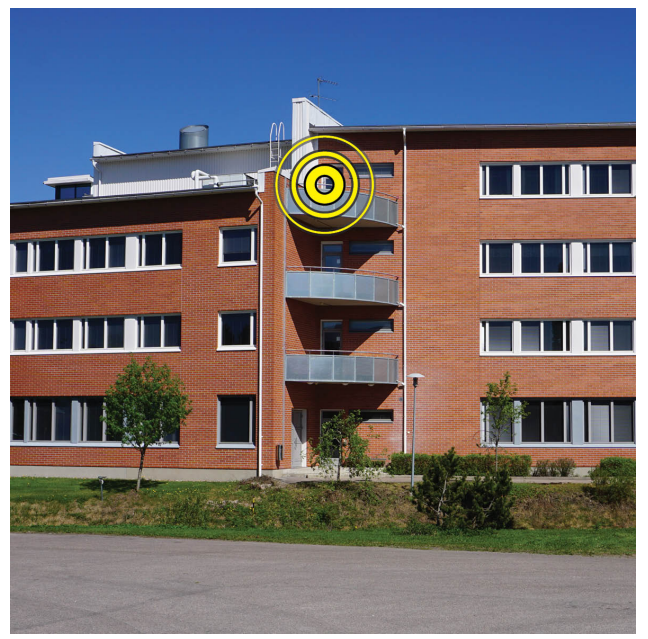

Figure 2. The RF source is located inside to the floor of building. The source is marked with yellow circles.

measurement time. In this interference measurement trial, flight operations durations were approximately 15 minutes.

The aviation regulation of UAS operation is taken care of during the trial. At the moment, UAS regulation varies between different countries [11]. This trial was performed in Finland according to existing Finnish Transport Safety Agency (Trafi) regulations [12].

UAV flights were performed in manual control. The UAS setup used in measurement is depicted in Figure 3. The ground stations were used for the flight and payload monitoring. The telemetry module of UAV was connected to the ground station. One $360^{\circ}$ camera was for flight and measurement monitoring purposes.

\section{Test Devices}

When test devices are planned for UAS measurement, the size and weight limitations have to be taken into account.

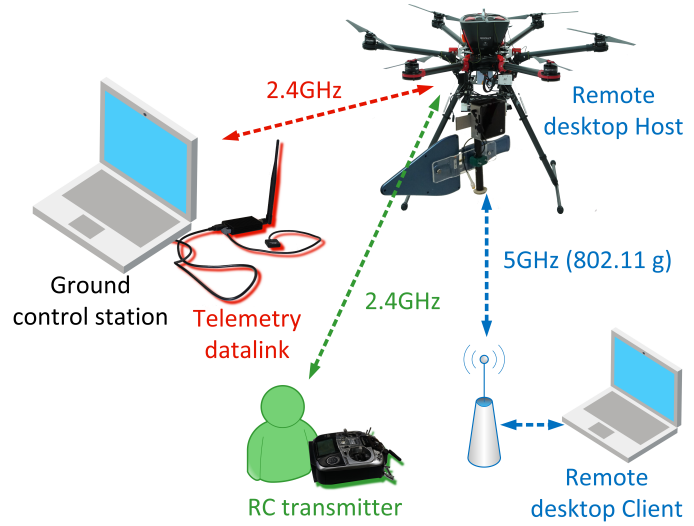

Figure 3. UAS Measurement setup in use.

Hand-held Aaronia Spectran HF-6105 was the spectrum analyser used in the measurement. It is suitable for measurements with UAS because of small size and low weight to minimize the take-off weight of UAS DJI S900. Frequency range is from $1 \mathrm{MHz}$ to $9.4 \mathrm{GHz}$, thus it can be used in all kinds of monitoring measurements on various frequency ranges. Aaronia MCS Spectrum Analyser Software was used to the real-time remote control of measurements. In this trial, Aaronia HyperLOG 60100 directional logarithmic-periodic antenna was used, it covers frequency range from $680 \mathrm{MHz}$ to $10 \mathrm{GHz}$.

\section{Measurement Method}

When authority gets an announcement of RF interference suspicion, the authority needs to find this RF interference source for elimination. The authority analyses the interference according to the announcement and performs spectrum measurements. The target is to locate the source of interference as accurately as possible. The normal procedure is to find interference with scanning larger area to find the building and the direction were the RF signal is originating. This approach was the starting point to develop interference locating process. The determining process of the RF interference source had two phases. The first phase was premeasurement with the approximate scanning of the environment. The premeasurement is described in the sub-section A. The second phase focuses on the measurement of the accurate location of the $\mathrm{RF}$ interference (described on the sub-section B). The accurate location determination phase was performed with UAS in the air and manually on the ground level. The ground level measurement (sub-section C) was a reference method for UAS measurement.

The same spectrum analyzer and the log antenna were used as a measurement system determining the power of an interfering signal in all measurements. On the air measurements the antenna, spectrum analyser and mini-pc were mounted at UAS. During the premeasurement scanning, radio spectrum power level is measured. Radio spectrum power level received by the antenna of a measurement system is referred to as background noise floor level. This noise floor level includes RF power from natural sources but also radiated interference 
TABLE II.

MEASUREMENT PARAMETERS

\begin{tabular}{|c|c|c|}
\hline Parameter & Value & Unit \\
\hline Center frequency & 864.850 & $\mathrm{MHz}$ \\
\hline Span & 1.00 & $\mathrm{MHz}$ \\
\hline RBW & 30 & $\mathrm{kHz}$ \\
\hline VBW & 30 & $\mathrm{~Hz}$ \\
\hline Attenuation & 0 & $\mathrm{~dB}$ \\
\hline Sample time & 5 & $\mathrm{~ms}$ \\
\hline
\end{tabular}

from radio transmitters of a different kind of communications networks and individual transmitters. The increased level of the noise floor on a given frequency range can reveal the location or existence of RF interference. The important is to define measurement parameters according to interference source frequency and power level. Parameters were defined in premeasurement. Measurement parameters are in the table II. Measurement results were collected in the log file. The waterfall view was used during the measurement to real time monitoring.

\section{A. Premeasurement}

When locating unknown RFI, the direction and polarization are not known and in some cases not even the frequency. The target of the premeasurements were to locate roughly where the RF source was placed. The premeasurements started with a triangle measurements at ground level, which is normal situation. The measurement setup was installed in three different locations (Fig. 1) and similar measurements were performed in all these locations. The measurement as done by scanning the receiving antenna in all directions. Based on these premeasurements, the study continued with inflight measurements with drone. The premeasurement revealed that vertical polarization was a dominating polarity for RF source. Based on this obsevations, the vertical polarization was used in all measurements.

The height of the flight was 15 meters, that is over the roof top level of surrounding buildings of the area where RF source was located. The RF source was located on a certain building. The first premeasurement flight was performed around the suspicion area. During the flight, the measurement antenna was pointing towards surrounding buildings. The UAS monitoring system was flown around the building to determine in which part of the building the RF noise was initiating. The flying route is shown in figure 1 . The waterfall view of the rotation measurement clearly showed that RF transmitter was located on the "south" side of the building. The next phase of premeasurement was performed on the south side of the building to scan the direction of the RF source between the two buildings. The UAS was turned around in one position with the same height and in the same coordinate so that antenna was scanning 360 degrees. This measurement gave the measurement location for reference measurements and vertical scanning.

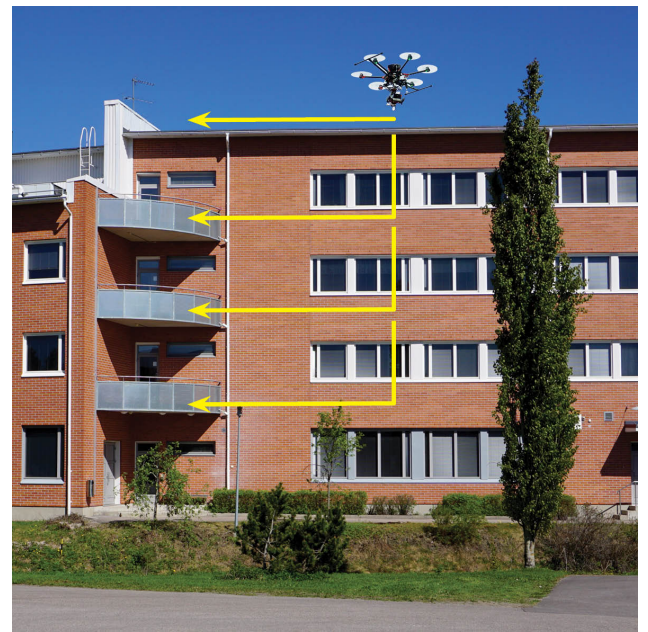

Figure 4. Yellow lines show flying heights of vertical scan measurement.

\section{B. Vertical Scan of the Building with the UAS}

The vertical scan measurement was performed in the geolocation that was defined in the premeasurement phase. This location was approximately at 45 meters distance from the building. During the measurement, only the height of measurement is changing. The UAS was scanning the building from ground to top by hovering at the height of each floor 25 seconds and then it moved to the next floor. Measurement was started from the second floor with height of 6 meters. Measurement were repeated from the third at 9 meters and the fourth at 12 meters and finally over the roof top level of the building at 16 meters (figure 4 ).

\section{Reference Measurement on the Ground}

Reference measurements were performed at 45 meters distance from the RF source on the ground level. This measurement location was determined on premeasurements having the strongest direction on the RF signal. The measurement antenna was pointed towards different floors to find out on which floor RF signal source was located (figure 5). The antenna was pointed towards different floors the same 25 seconds measurement time as was used in UAS measurement.

\section{Results}

The aim of the study was to clarify whether there are differences in the capability of finding the interference source from the ground or air. In this case the results of reference measurement on the ground level with the results of measurements on the air were compared. The comparisons are performed using the numerical values as well as the visual waterfall format. The waterfall view of the spectrum analyser was used for visual comparison because it gives the good indication of time-based signals. The waterfall view is a way to show measurement data as a heat map in frequency (x-axis) and time (y-axis) domain. It also makes easier to compare instantaneously changes on the signal level during the whole measurement. 


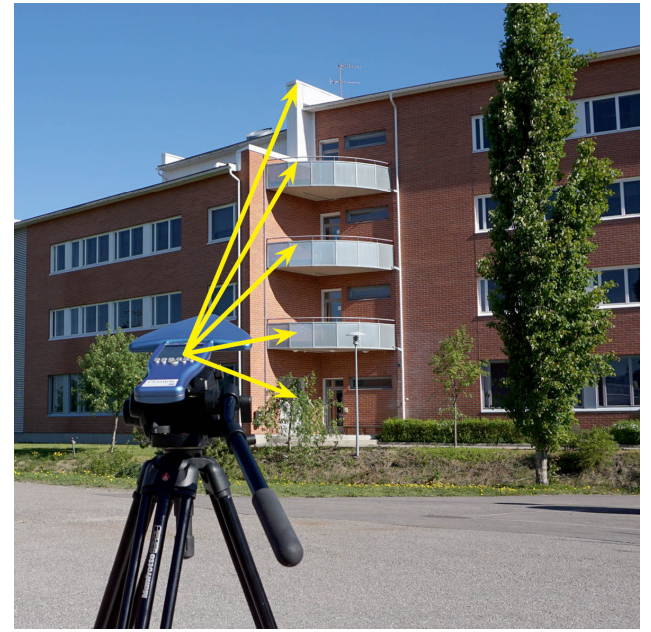

Figure 5. Yellow lines show direction of receiving antenna at the reference measurement.

Figure 6 presents the waterfall view of the reference ground level measurement. The red lines are added to waterfall view to clarify change in the antenna direction. The red line shows on which floor antenna is pointed. Measurement starts from the lowest red line when the measurement antenna is pointed out towards the first floor. The second red line is correspondingly showing were measurement starts toward second floor. The waterfall view does not clearly reveal the location of the RF source.

Table III shows the average values of the signal strength measurement on the ground. Results are analysed in boxplots (Figure 7 and 9). The box presents the interquartile range (IQR) between 25 and 75 percentile. The median (black line) and mean (orange dot) values are marked inside the box. The end of whiskers shows the minimum and maximum values, outliers are marked with asterisks. The highest signal level $60.8 \mathrm{dBm}$ was measured above the building even the signal source was located on the fourth floor. The lowest signal level was measured from the ground floor $-63.9 \mathrm{dBm}$. The variation between signal levels was $3.1 \mathrm{~dB}$.

Changes on the signal level in the UAS measurement is shown in Figure 8. Red lines are added in this waterfall view similarly than to reference measurement waterfall view. In this case UAS transition between floors and time are marked with red lines as well as periods when UAS has hovered for the actual measurement. The waterfall view shows clearly that RF source is located between 9 and 15 meters. Average and variation of results from the stable measurement periods are gathered to Table IV and figure 9. These are average values of results on each measurement height. The lowest value -58.76 $\mathrm{dBm}$ is over the building. The highest signal level $-53.49 \mathrm{dBm}$ was founded at the fourth floor where the signal source was. The variation between signal levels was $5.3 \mathrm{~dB}$.

Comparison between reference measurement on the ground and UAS measurement revealed that location of the signal source can be determined more accurately with UAS. When

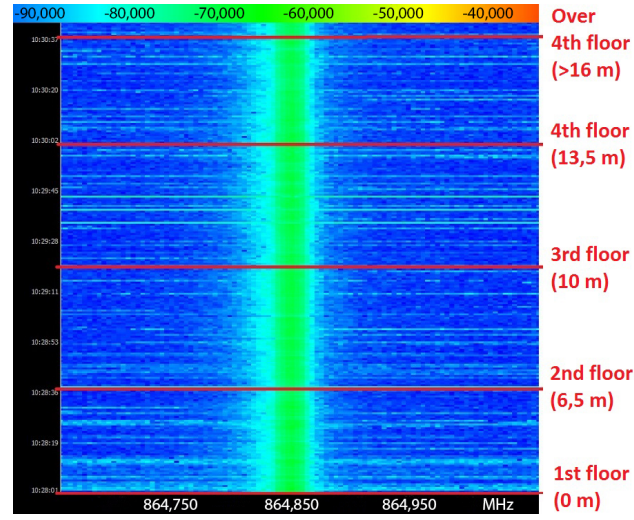

Figure 6. Waterfall view of results measured from the ground level

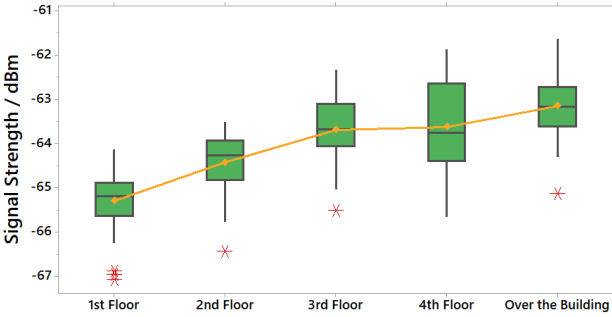

Figure 7. The boxplot figure of the ground measurement

TABLE III. MEASUREMENT RESULTS ON THE GROUND

\begin{tabular}{|c|c|c|}
\hline Floor & Angle & Signal strength $/ \mathbf{d B m}$ \\
\hline $1^{\text {st }}$ & 0 & -63.9 \\
\hline $2^{\text {nd }}$ & 7.6 & -63.4 \\
\hline $3^{\text {rd }}$ & 12.5 & -62.0 \\
\hline $4^{\text {th }}$ & 17.3 & -61.5 \\
\hline Over the building & 19.6 & -60.8 \\
\hline
\end{tabular}

the target is to locate RF signal from the high build, the materials of the building will attenuate the signal. All structures like floors, roofs, walls and windows affect the RF signal. The metal balcony and railing most probably attenuated RF signal. In the reference measurement the level of the measured RF signal seemed to be almost the same in spite of which floor the antenna was directed to. However slightly higher levels are detected when the antenna is directed towards upper floors.

\section{CONClusion ANd Future Work}

In this study UAS was used in two phases to locate RF source which simulated RF inference. The UAS equipped with a suitable spectrum analyzer and antenna scanned four floor flat on all sides to determine the building side were RF source was located. Thereafter, in the second phase, the UAS with RF measurement setup was used to the vertical scan of building to find the strongest radiation direction. The second phase measurement results from ground based and UAS based measurements were compared. The comparison revealed that the new UAS based measurement method was able to determine the location of the RS source more accurately than existing methods. With four floor building, it was 


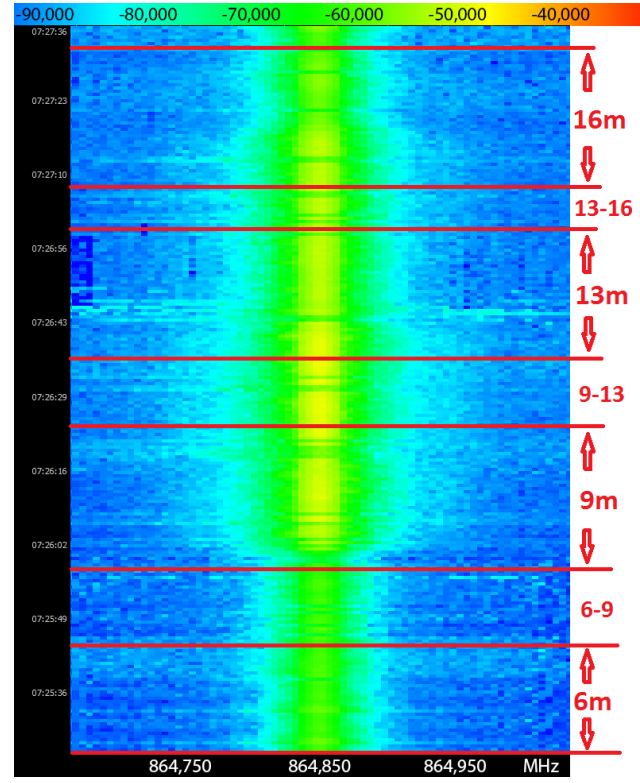

Figure 8. Waterfall view of results measured from the aerial measurement

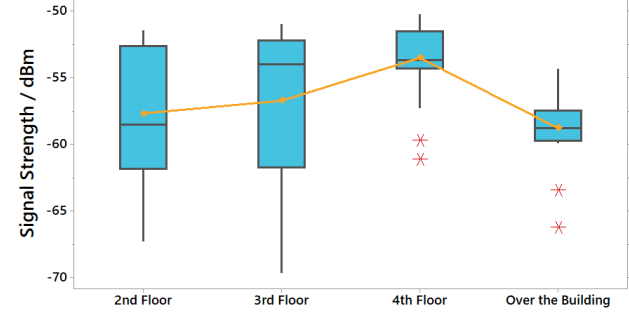

Figure 9. The boxplot figure of the aerial measurement

TABLE IV. MEASUREMENT RESULTS ON THE AIR

\begin{tabular}{|c|c|c|}
\hline Floor & Height (meters) & Signal strength $/ \mathbf{d B m}$ \\
\hline $1^{\text {st }}$ & - & - \\
\hline $2^{\text {nd }}$ & 6 & -57.12 \\
\hline $3^{\text {rd }}$ & 9 & -56.69 \\
\hline $4^{\text {th }}$ & 12 & -53.49 \\
\hline Over the building & 16 & -58.76 \\
\hline
\end{tabular}

able to determine the floor of the RF source with accuracy of two floors from observing the waterfall results and to the accurate floor if we trust (allow $3 \mathrm{~dB}$ diffrerence to distinguish between floors) the measurement results in Table IV. Based only on the ground floor measurements it was impossible to determine the height of RF source. This study showed that using UAS for determining the location of interference has benefits. The height and the floor of the interfering device can be defined much more accurately and quickly compared to existing methods (hand-held devices and monitoring van).

The conclusion is that UAS with RF measurement setup suits national authorities as a tool for airborne mobile monitoring. UAS can help locating the direction of the most powerful signals of the interference source. The use of UAS could be useful especially in the challenging urban environment. Future studies will focus on further development the measurement and visualization of the UAS usage in the RF measurements, e.g. look at the effect of increasing directivity (antenna selections) and the assessment of performance in triangulation (radio direction finding). A 3D visualization of the measurement results is important during the analyzing phase. There is also a need to develop automated measurement methods since there will be more and more competitive wireless systems. This will challenge interference-free spectrum usage and this will require more monitoring activities in the field. A further notion on this type of technology is the possibility to use it on real time network planning by network operators of e.g. 5G networks where nomadic or mobile network elements are foreseen as well as proactive mobility management by identifying user service gaps or "holes" in the network.

\section{ACKNOWLEDGEMENT}

This work has been carried out in the framework of the IMAGE 5G project. The authors would like to acknowledge IMAGE 5G research consortium that consist of Centria University of Applied Sciences, Nokia, PehuTec Oy, Siipotec Oy, Pohjonen Group Oy, The Finnish Transport and Communications Agency, Finnish meteorological institute and Business Finland - The Finnish Funding Agency for Innovation.

\section{REFERENCES}

[1] Ericsson, "Mobile data traffic growth outlook." [Online]. Available: https://www.ericsson.com/en/mobility-report/reports/june-2018/ mobile-data-traffic-growth-outlook

[2] A. Osseiran, J. F. Monserrat, P. Marsch, 5G Mobile and Wireless Communications Technology. Cambridge University Press, 2016.

[3] Finnish Transport and Communications Agency Traficom, "Radio frequency regulation." [Online]. Available: http://finlex.fi/fi/ viranomaiset/normi/480001/44839

[4] Viestintävirasto, "Radiohäiriöiden kohteet ja aiheuttajat 2014." [Online] Available: https://https://www.viestintavirasto.fi/tilastotjatutkimukset/ katsauksetjaartikkelit/2015/radiohairioidenkohteetjaaiheuttajat2014.html

[5] The Electronic Communications Committee of the European Conference of Postal and Telecommunications Administrations, "Fm 22 summary of the annual interference statistics questionnaire for reported cases in 2017." [Online]. Available: https://www.cept.org/ecc/groups/ecc/wg-fm/ fm-22/client/introduction/

[6] infoDev and the International Telecommunications Union (ITU), "Ict regulation toolkit / 5.6 spectrum monitoring and compliance." [Online]. Available: http://www.ictregulationtoolkit.org/toolkit/5.6

[7] S. Duthoit, J. L. Salazar, W. Doyle, A. Segales, B. Wolf, C. Fulton, and P. Chilson, "A new approach for in-situ antenna characterization, radome inspection and radar calibration, using an unmanned aircraft system (UAS)," in 2017 IEEE Radar Conference (RadarConf), May 2017, pp. 0669-0674.

[8] M. García-Fernández, Y. Á. López, A. Arboleya, B. González-Valdés, Y. Rodríguez-Vaqueiro, M. E. D. C. Gómez, and F. L.-H. Andrés, "Antenna diagnostics and characterization using unmanned aerial vehicles," IEEE Access, vol. 5, pp. 23 563-23 575, 2017.

[9] M. Heikkilä, J. Erkkilä, M. Koskela, J. Heikkilä, T. Kupiainen, J. Tervonen, and M. Migliore, "Development of the measurement method for challenging nlos conditions in mobile networks," in 2017 IEEE International Workshop on Measurement and Networking (M N), Sept 2017, pp. 1-6.

[10] ANFR, "Spectrum monitoring with drones." [Online]. Available: https://www.cept.org/

[11] DroneRules.eu, "Drone regulations in EU, Norway and Switzerland." [Online]. Available: http://dronerules.eu/

[12] Liikenteen turvallisuusvirasto, "Trafi unmanned aviation regulation OPS M1-32." [Online]. Available: https://www.trafi.fi/filebank/ a/1483970125/4a6ac53bf4b1cb434d7f85a15f36dde0/23661-OPS_ M1-32_RPAS_2016_eng.pdf 